\title{
Modeling and Analysis of Disease-Induced Host Growth in the Epidemiology of Take-All
}

\author{
D. J. Bailey and C. A. Gilligan
}

First author: INRA-Bordeaux, UMR Santé Végétale, BP81, 33883 Villenave d'Ornon; and second author: Department of Plant Sciences, University of Cambridge, Downing Street, Cambridge, CB2 3EA, UK. Accepted for publication 8 January 2004.

\begin{abstract}
Bailey, D. J., and Gilligan, C. A. 2004. Modeling and analysis of diseaseinduced host growth in the epidemiology of take-all. Phytopathology 94:535-540.

Epidemiological modeling, together with parameter estimation to experimental data, was used to examine the contribution of disease-induced root growth to the spread of take-all in wheat. Production of roots from plants grown in the absence of disease was compared with production of those grown in the presence of disease and the precise form of diseaseinduced growth was examined by fitting a mechanistic model to data describing change in the number of infected and susceptible roots over time from a low and a high density of inoculum. During the early phase of the epidemic, diseased plants produced more roots than their non-

infected counterparts. However, as the epidemic progressed, the rate of root production for infected plants slowed so that by the end of the epidemic, and depending on inoculum density, infected plants had fewer roots than uninfected plants. The dynamical change in the numbers of infected and susceptible roots over time could only be explained by the mechanistic model when allowance was made for disease-induced root growth. Analysis of the effect of disease-induced root production on the spread of disease using the model suggests that additional roots produced early in the epidemic serve only to reduce the proportion of diseased roots. However, as the epidemic switches from primary to secondary infection, these roots perform an active role in the transmission of disease. Some consequence of disease-induced root growth for field epidemics is discussed.
\end{abstract}

It is widely acknowledged that disease affects the growth of infected plants but it does so in ways that are dynamic, often subtle, and that are difficult to quantify and understand. Most attention to date has been focused on the relationship between disease and loss of economic yield. Here, however, we focus on how diseaseinduced changes in host growth controls the availability of susceptible tissue and, hence, the rate of spread of infection and disease through the host population. The unit of population may be a leaf, stem, or root, depending on the pathogen. Subtlety in epidemic dynamics arises because a small amount of infection may stimulate the host to overcompensate by producing more healthy tissue than is lost to disease $(4,7)$. Undercompensation follows as the disease load on the plant increases and the plant is unable to offset the loss of healthy tissue. We have shown previously that each of these affects the dynamics of epidemics in different ways $(4,5)$. Although overcompensation early on in the epidemic dilutes the proportion of infected tissue, it does not necessarily follow that it slows the spread of disease. Instead, the local surfeit in susceptible tissue may help to speed up the epidemic as the pathogen uses the newly produced leaves or roots as a dispersal bridge to reach more distant tissue. As the host ages and the epidemic progresses, the median distance between infected and susceptible tissue increases within and between plants and the epidemic may slow down (1). Experimental support comes from amputation studies in which tissue is removed to simulate disease and the resulting growth of tissue is monitored (11). Simple experimental comparisons of leaf and root growth in diseased and control (disease-free) plants $(3,8,12)$ also reveal the effects of disease, but these studies only describe effects without identifying the underlying causal dynamical relationships. This requires a combination

Corresponding author: D. J. Bailey; E-mail address: dbailey@bordeaux.inra.fr

Publication no. P-2004-0323-01R

(c) 2004 The American Phytopathological Society of a theoretical framework with experimentation to differentiate the effects of disease-induced host growth and transmission dynamics on epidemic spread $(4,5)$. Thus, we take a simple $S I$ model for the flow of susceptible $(S)$ tissue into the infected class $(I)$, (where $N=I+S$ is the total number of stems, roots or leaves) giving

Susceptible $\quad \frac{\mathrm{d} S}{\mathrm{~d} t}=g(N)-f(I, S)-\lambda(I) S$

Infected

$$
\frac{\mathrm{d} I}{\mathrm{~d} t}=\lambda(I) S
$$

in which $\lambda(I)$ is the force of infection by which susceptible roots, leaves, or stems become infected and move into the infected class. The typical form of the force of infection is given by $\lambda(I) S=\beta S I$ for simple mass action transmission (4), in which $\beta$ is the transmission rate for infection and disease. Host growth is separated into two components, one for intrinsic growth $[g(N)]$, typified by a logistic function, and the other for disease-induced host growth $[f(I, S)]$. Depending on the sign of $f(I, S)$, disease-induced host growth may lead to over- or undercompensation. Some progress has been made in the mathematical analysis (6) of the model in equation 1, but experimental testing is embryonic (5).

Here we extend the approach to consider the infection of wheat roots by the take-all fungus, Gaeumannomyces graminis var. tritici. We choose this because of its economic importance and previous experimental work that has suggested a flexible response of wheat in the production of main adventitious roots to infection load (12). The trajectory of a take-all epidemic caused by G. graminis depends on the interaction between inoculum and the growing root system. As with most epidemics, it can be divided conveniently into consecutive phases dominated by primary infection and secondary infection $(1,3)$. Disease is initiated by primary infection from particulate inoculum in the soil and, depending on the rate of decay of particulate inoculum, may be largely restricted to the seminal roots. Having infected the seminal roots, disease continues to spread by secondary root-to-root infection, stimulated 
by the production of adventitious roots (1). We use a combination of mathematical modeling and experimentation to examine both the effect of disease on root production and then the contribution of disease-induced root growth on the spread of disease. Data for the spread of disease, decay of particulate inoculum, and root production are obtained from microcosm experiments. Specifically we ask, (i) does take-all affect the production of wheat roots; (ii) is there evidence for both stimulation and inhibition of root production; and (iii) how does this affect, in turn, phases of primary and secondary infection? We use the generic model given in equation 1 and compare the goodness-of-fit with three different forms of the host response to infection load. These are (i) the absence of disease-induced root growth, (ii) stimulation of root production when few roots are infected and inhibition of root production when the number of infected roots passes a threshold, and (iii) stimulation and inhibition of root production (as described) where the level of the response increases with the number of susceptible healthy roots. Finally, to examine the contribution of disease-induced root growth on the spread of disease, we use the model and parameter estimates from model fitting to simulate and compare epidemics in the presence and absence of the response.

\section{THEORY AND APPROACHES}

Model derivation. The model in equation 1 is adjusted to allow for dual sources of infection, with decay of primary inoculum in soil and an assumption of logistic growth for $g(N)$,

$\begin{array}{lll}\text { Susceptible roots } & \frac{\mathrm{d} S}{\mathrm{~d} t}=b N \frac{(\kappa-N)}{\kappa}-\left(r_{p} X+r_{s} I\right) S-f(I, S) \\ \text { Infected roots } & \frac{\mathrm{d} I}{\mathrm{~d} t}=\left(r_{p} X+r_{s} I\right) S \\ \text { Particulate inoculum } & \frac{\mathrm{d} X}{\mathrm{~d} t}=\left(-r_{d} X\right)\end{array}$

The intrinsic production of new roots increases sigmoidally during the period of the experiment where $\kappa$ is the maximum number of roots per plant, $b$ is the per capita rate of production of susceptible roots, and $N$ is the total number of roots at time, $t$ (i.e., $N=I+S)$. Particulate inoculum $(X)$ decays exponentially over time with a rate, $r_{d}$. The parameters $b$ and $\kappa$ are estimated from empirical data for root growth in the absence of disease in order to give a measure of intrinsic root growth, while $r_{d}$ is estimated from a contemporary experiment to measure the decay of inoculum over time.

We could have examined the effects of disease on root growth by examining changes in the parameters $b$ and $\kappa$ of the growth term

$$
b N\left(\frac{\kappa-N}{\kappa}\right)
$$

Instead, in keeping with the model given in equation 1 , we used $f(I, S)$ to describe disease-induced root production explicitly while $b$ and $\kappa$ were fixed according to the estimates from disease-free controls. We examined the performance of three forms of the model with varying forms of disease-induced root production, $f(I, S)$,

$$
\begin{gathered}
f(I, S)=0 \\
f(I, S)=\frac{\left(\alpha_{1} I^{2}-\alpha_{2} I\right)}{\left(\alpha_{3}+I^{2}\right)} \\
f(I, S)=\frac{\left(\alpha_{1} I^{2}-\alpha_{2} I\right)}{\left(\alpha_{3}+I^{2}\right)} S
\end{gathered}
$$

(Model 1)

(Model 2)

Model 1 assumes no effect of disease on root production. Model 2 allows for stimulation of root production when the number of infected roots is low and inhibition of root production when the number exceeds a critical level. Model 3 includes a per capita response to disease in which we assume that stimulation of root growth is proportional to the number of susceptible roots. The models were fitted to change in the numbers of infected and susceptible roots over time using Facimile (version 1.5.11, AEA Technology, Harwell, UK). Parameter estimates for $r_{p}, r_{s}$, and where applicable, $\alpha_{1}, \alpha_{2}$, and $\alpha_{3}$ were estimated and the goodness-of-fit of the models was compared by examining residual sums of squares, trends in residuals, and the biological plausibility of parameter estimates for treatments with different initial densities of inoculum. The parameters $r, \kappa$, and $r_{d}$ were fixed at the values for the disease-free controls.

To assess the effect of disease on root production, we examined curves describing (i) differences between intrinsic (control plants) and induced (infected plants) root growth over time and (ii) change in $f(I, S)$ with increase in numbers of infected roots.

While it is possible to test the effect of disease on root production experimentally by comparing the dynamics of root production in the presence and absence of disease, it is not possible to examine the contribution of disease-induced root growth to the spread of disease by experiment alone. To do this would require comparison of two cultivars in the presence of disease that differed only in response to disease. Instead, the model and parameter estimates obtained from fitting to disease data were used to simulate epidemics in the presence and absence of disease-induced root growth.

Experimentation: Disease progress curves. Data for disease dynamics and host growth were obtained by growing plants in sand microcosms consisting of lengths of layflat tubing (Layflat Tubing, Isle of Wight) measuring $250 \mathrm{~mm}$ in length and $100 \mathrm{~mm}$ wide. The tubing was stapled at one end and filled with $1,000 \mathrm{~g}$ of sand (grade 16/30; Hepworth Minerals and Chemicals Ltd., Redhill, UK). A nutrient solution (Hoagland's) was added to achieve a gravimetric water content of $10 \%$ by weight. Each microcosm was planted with a pregerminated seed of winter wheat cv. Savannah. Microcosms were incubated at $17^{\circ} \mathrm{C}$ with $16 \mathrm{~h}$ light and grown on an incline of $30^{\circ}$ from the vertical to guide roots to the rear of the pack, allowing for nondestructive observation and quantification of healthy and infected roots over time. Inoculum was prepared by removing disks of colonized potato dextrose agar from the growing edge of a 7-day-old culture of G. graminis var. tritici (IMI 389305). After 6 days, either a single inoculum unit (low inoculum density) or four inoculum units (high inoculum density) were introduced randomly into microcosms at a depth of $100 \mathrm{~mm}$. There were 10 replicate microcosms for each of three treatments: no inoculum, low density of inoculum, and high density of inoculum. A visual, nondestructive assessment of the number of diseased (stelar discoloration) and disease-free roots was made each week in each microcosm following inoculation for 10 consecutive weeks. Soil packs were maintained at constant weight by adding Hoagland's solution.

Inoculum decay. To estimate the rate of inoculum decay, $r_{d}$, and the duration of primary infection, the decay in infectivity of inoculum was examined independently during the first 5 weeks of the experiment. Six units of inoculum were placed in each of 10 sand microcosms at the beginning of the experiment. After 0, 6, $10,14,18,28$, and 35 days, a single unit of inoculum was removed from each microcosm and placed against the seminal roots of a 7-day-old wheat plant (cv. Savannah). The sand packs were incubated under the conditions described previously and assessed for disease after 21 days to provide estimates for change in the proportion of infective inoculum units with time.

\section{RESULTS}

Disease dynamics. At a low initial density of inoculum, the number of infected roots increased gradually for the first 35 days after which there was a sharp increase in the rate of infection that 
was maintained for the remainder of the experiment. During the same period, the number of susceptible roots increased to a maximum at around 35 days and then decayed asymptotically toward zero (Fig. 1A). The combination of these processes resulted in a curve describing the proportion of infected roots that increased during the first 20 days to an initial plateau of 0.3 and then increased again after 35 days toward a final plateau of $\geq 0.9$ after 77 days (Fig. 1C). Increasing the inoculum density increased the rate of infection during the initial 35 days but not during the period thereafter (Fig. 1B). This increased the height of the initial plateau for change in the proportion of infected roots over time to 0.4 (Fig. 1D). The bioassay for inoculum decay showed that the probability of infection from particulate inoculum decayed rapidly during the course of the experiment from 1.0 at day 0 to below 0.01 after 24 days where $r_{d}$ was estimated as 0.20 (with lower and upper $95 \%$ confidence limits of 0.163 to 0.237 ).

Intrinsic root growth. In the absence of disease, the number of main root axes increased exponentially over time. The maximum number of roots per plant, $k$, and the intrinsic growth rate, $b$, were estimated as 32.6 (17.8 to 59.7) and 0.031 (0.014 to 0.065), respectively.

Disease-induced root growth. To examine the effect of disease on root growth, we compared curves describing differences in the total numbers of roots produced by infected and disease-free plants over time (Fig. 2). At low inoculum density, the average number of additional roots produced by infected plants increased to a maximum of two after 50 days and then decreased to less than one additional root after 77 days. At high inoculum density, change in the number of additional roots followed a similar trend, but by the end of the experiment, disease-free plants had produced more roots than their infected counterparts.

We compared the goodness-of-fit of models 1 through 3 (Fig. 1 ; Table 1) to changes in infected and susceptible roots over time. The parameters $b$ and $k$ for intrinsic root growth were estimated from root growth in the absence of disease, while the decay of

Low inoculum density
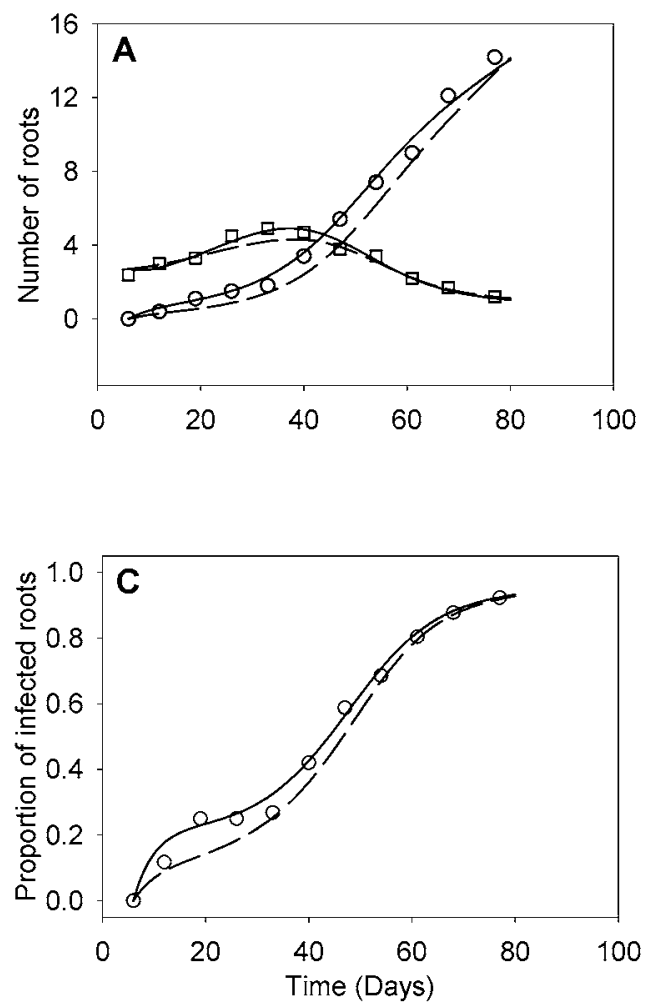

inoculum, $r_{d}$, was estimated from a separate experiment described previously. Hence, $b, k$, and $r_{d}$ were treated as fixed in estimating the epidemiological parameters $r_{p}, r_{s}, \alpha_{1}, \alpha_{2}$, and $\alpha_{3}$. Model 1 consistently underestimated the numbers of infected and susceptible roots at low inoculum density (Fig. 1A) and the number of infected roots until day 70 at high inoculum density (Fig. 1B). This resulted in underestimation of the proportion of infected roots over time (Fig. 1C). Model 2, allowing for stimulation of root growth at low levels of disease and inhibition of growth at high disease levels, significantly improved the goodness-of-fit to change in infected and susceptible roots (Fig. 1A and B; Table 1) and the proportion of infected roots (Fig. 1C and D) over time. Model 3, which allowed for increased stimulation when more

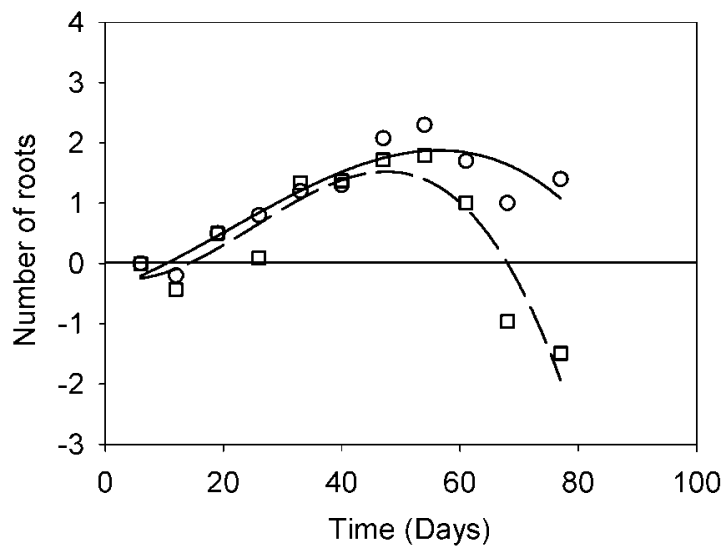

Fig. 2. Difference between the average number of roots on plants infected with either a low (open circles) or high (closed circles) density of inoculum and their uninfected counterparts for the duration of the take-all epidemic. Intrinsic root growth in the absence of disease, $b$, over time, $t$, was described by the function $b=2.73 \cdot \exp (0.0216 t)$.
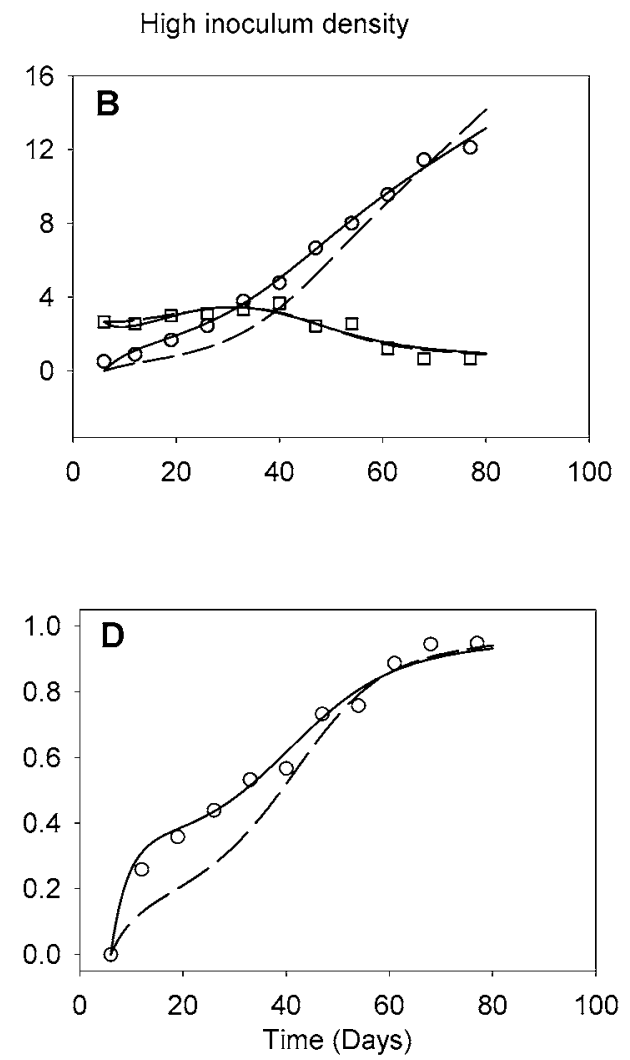

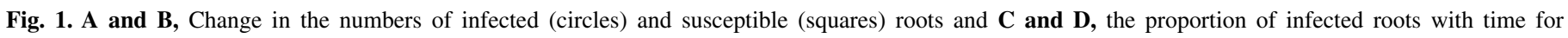
epidemics of take-all from a low or a high density of particulate inoculum. Data fitted with model 1 (dotted line) and model 2 (solid line) are shown. 
susceptible, healthy roots were present, did not improve the fit (Table 1).

Parameter estimates of $\alpha_{1}, \alpha_{2}$, and $\alpha_{3}$, obtained from model fitting, were used to examine the effect of inoculum density on the host response as a function of infected roots. Inoculum density did not significantly affect estimates of $\alpha_{1}, \alpha_{2}$, and $\alpha_{3}$ (Table 1 ), and consequently, curves describing $f(I)$ were very similar (Fig. 3). Note that increase and decrease in the rates of root production are reflected by negative and positive values of $f$, respectively. The response curves of $f(I)$ indicate that stimulation of root production increases to a maximum when the number of infected roots is close to one and declines to zero as the number of infected roots approaches four. Thereafter, $f(I)$ becomes positive, consistent with inhibition of root production.

Although the response curve, $f(I)$, was not affected by inoculum density, the initial conditions, mediated by inoculum density, do affect the change in the level of the response over time. As the epidemic progresses, the number of infected roots and therefore the course of $f(I)$ over time is displaced for epidemics initiated from different levels of inoculum (Fig. 4). During the period of primary infection (between 6 and 35 days) and at low inoculum density, $f(I)$ remains negative, suggesting stimulation of root production (Fig. 4). After 40 days, $f(I)$ became positive and root production was inhibited. Increasing inoculum density did not affect the amplitude of stimulation during primary infection but reduced the duration of stimulation, inhibiting root production shortly after 30 days.

Simulation of the effect of disease-induced root growth on the spread of disease. The parameters values for $r_{p}, r_{s}, \alpha_{1}, \alpha_{2}$, and $\alpha_{3}$, obtained by fitting model 2 to change in infected and susceptible roots over time, were used to simulate and compare the dynamics of disease in the absence and in the presence of diseaseinduced root growth (Fig. 5). During primary infection and at low inoculum density, disease-induced root growth increased the number of susceptible roots with little impact on infected roots (Fig. 5A). This resulted in a marginal reduction in the proportion of infected roots (Fig. 5C). However, during secondary infection, numbers of susceptible roots were ultimately reduced. This, combined with a significant increase in numbers of infected roots (Fig. 5A), leads to a large increase in the proportion of infected roots (Fig. 5C). The same trends were repeated for epidemics initiated from a high density of inoculum (Fig. 5B and D).

Increasing the amplitude and duration of the response did not increase the number of infected roots but led to a significant and sustained increase in the number of susceptible roots for epidemics dominated by primary infection (Fig. 6A). A rise in the number of susceptible roots was also seen for epidemics dominated by secondary infection. However, this was only transitory, these additional roots leading ultimately to an increase in the number of infected roots (Fig. 6B).

\section{DISCUSSION}

We have introduced and tested models for disease-induced root production in the epidemiology of take-all using a combination of simple epidemiological models and parameter estimation for data on disease dynamics from microcosm experiments. The models encompass epidemiological terms for primary infection, secondary infection, and inoculum decay in a simple $S I$ framework for changes in the numbers of susceptible $(S)$ and infected or diseased $(I)$ seminal and adventitious roots per plant. By partitioning the production of new susceptible roots on a diseased plant into terms for the intrinsic root growth and disease-induced root growth, we have shown that the dynamical change in the numbers of diseased and susceptible roots over time could only be explained by the mechanistic models when allowance was made for

TABLE 1. Estimates of parameters together with 95\% upper and lower confidence limits and residual sums of squares (RSS) from fitting models 1 through 3 to data describing the disease dynamics of take-all on winter wheat cv. Savannah at low and high inoculum density (ID)

\begin{tabular}{|c|c|c|c|c|c|c|c|}
\hline \multirow[b]{2}{*}{ Model $^{\mathrm{a}}$} & \multicolumn{5}{|c|}{ Parameters $\pm 95 \%$ confidence limits } & \multirow[b]{2}{*}{ RSS } & \multirow[b]{2}{*}{ df } \\
\hline & $r_{p}$ & $r_{s}$ & $\alpha_{1}$ & $\alpha_{2}$ & $\alpha_{3}$ & & \\
\hline \multicolumn{8}{|l|}{ Low ID } \\
\hline 1 & $0.025(0.017-0.035)$ & $0.017(0.016-0.019)$ & - & - & - & 9.07 & 20 \\
\hline 2 & $0.056(0.041-0.077)$ & $0.013(0.012-0.015)$ & $0.113(0.067-0.191)$ & $0.384(0.196-0.753)$ & $4.400(1.641-11.8)$ & 2.78 & 17 \\
\hline 3 & $0.11(0.073-0.177)$ & $0.0098(0.0079-0.012)$ & $0.089(0.051-0.155)$ & $0.311(0.152-0.637)$ & $6.39(2.32-17.6)$ & 9.39 & 17 \\
\hline \multicolumn{8}{|l|}{ High ID } \\
\hline 1 & $0.012(0.0081-0.017)$ & $0.021(0.0187-0.023)$ & - & - & - & 14.1 & 20 \\
\hline 2 & $0.038(0.0253-0.057)$ & $0.014(0.0116-0.017)$ & $0.108(0.051-0.227)$ & $0.37(0.145-0.945)$ & $3.917(0.65-23.6)$ & 8.54 & 17 \\
\hline 3 & $0.031(0.021-0.046)$ & $0.016(0.0136-0.0186)$ & $0.314(0.048-2.045)$ & $1.44(0.182-11.3)$ & $10.4(8.5-1,272)$ & 8.13 & 17 \\
\hline
\end{tabular}

${ }^{\text {a }}$ Model 1, no response; model 2, stimulation/inhibition; and model 3, stimulation/inhibition with per capita response for susceptible roots.

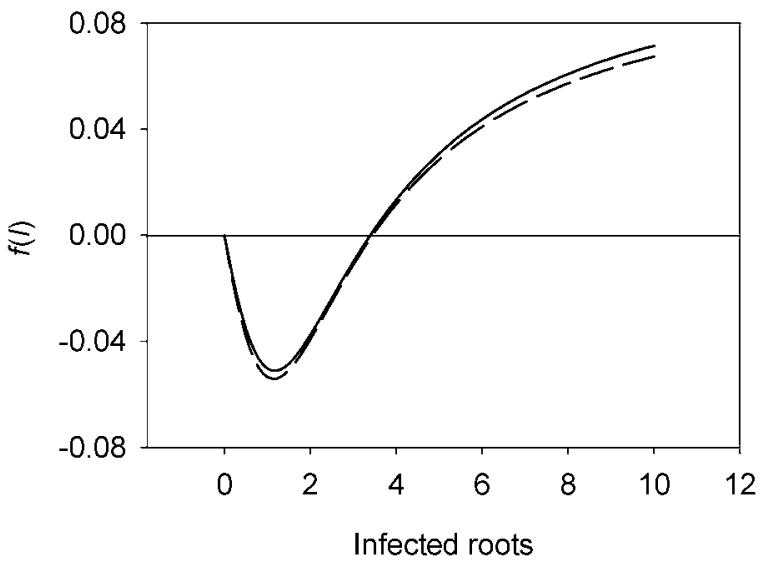

Fig. 3. Response curves for change in disease-induced host growth, $f$, with numbers of infected roots where $f=\left[\left(\alpha_{1} I^{2}-\alpha_{2} I\right) /\left(\alpha_{3}+I^{2}\right)\right]$ for a low (solid line) and a high (dotted line) density of particulate inoculum.

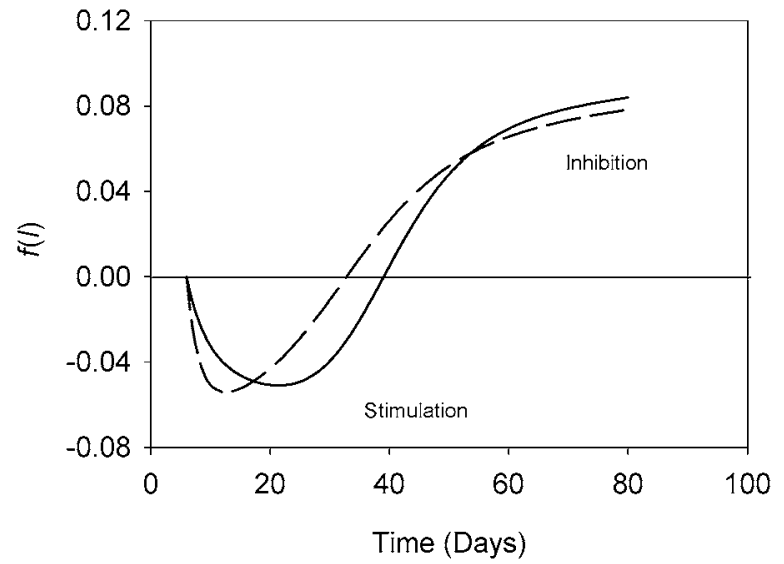

Fig. 4. Change in disease-induced host growth, $f$, with time, $t$, for epidemics initiated from a low (solid line) or high (broken line) density of inoculum. 
disease-induced root growth. More importantly, we detected a stimulatory-inhibitory response of root production to dynamical changes in the levels of disease roots per plant. This implies that when levels of disease within a plant are low, plants initially overcompensate in replacing diseased roots. This is followed by an inhibitory response as the plant fails to compensate for greater loss of diseased roots (Figs. 3 and 4).

The shape of this response was characterized by a curve relating the growth response $[f(I, S)]$ to the numbers of infected roots per plant $(I)$. Within the limited range tested, the relative amount of initial inoculum density did not affect the shape of the response (Fig. 3; Table 1) but it did affect the magnitude of the response during the course of the epidemic. High levels of initial inoculum caused high incidences of primary infection, which meant that maximum stimulation of root production was achieved after only 12 days and lasted for about 35 days (Fig. 4). A lower density of initial inoculum delayed the timing of the maximum response and extended the duration of stimulation to about 40 days (Fig. 4). The amplitude and duration for which the response remains stimulatory dictates the number of additional roots produced by
Low inoculum density

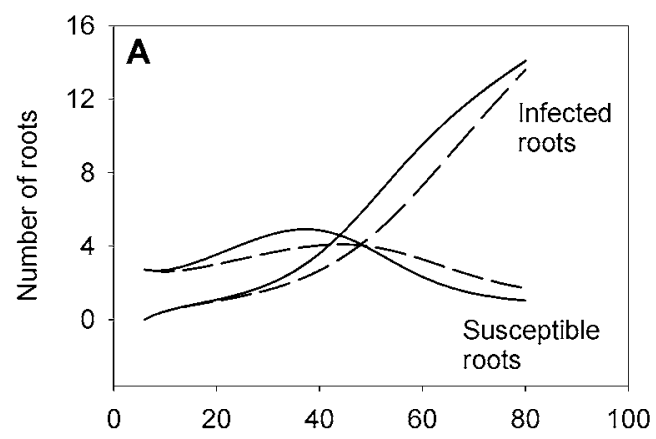

High inoculum density

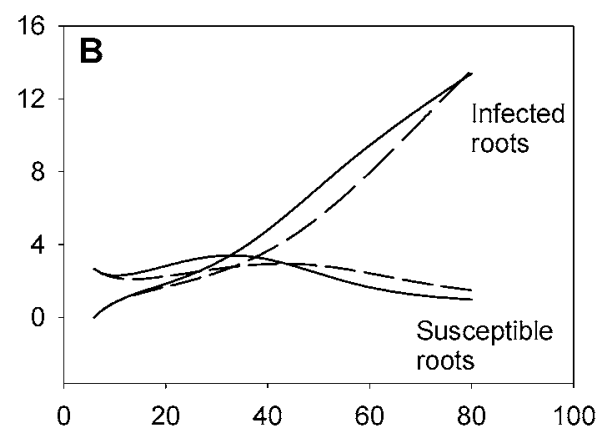

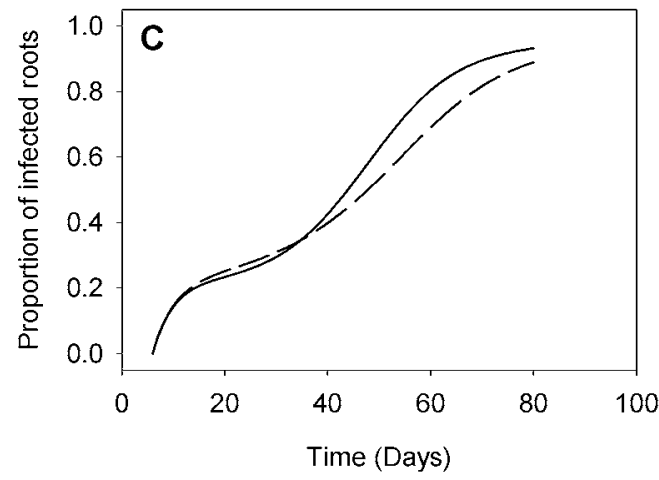

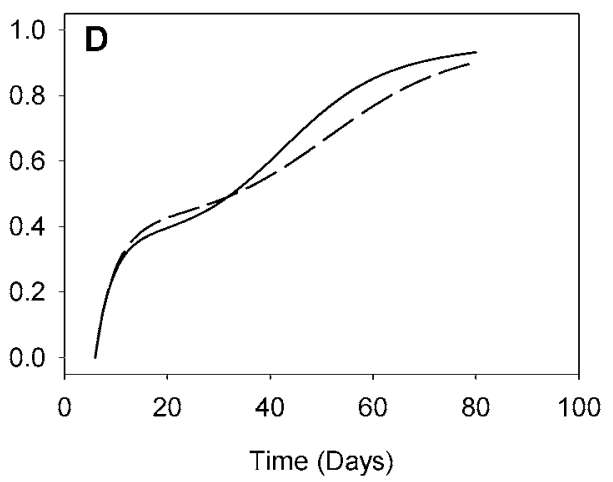

Fig. 5. The effect of disease-induced root growth on change in the number of infected and susceptible roots and the proportion of infected roots over time for epidemics from a $\mathbf{A}$ and $\mathbf{C}$, low or $\mathbf{B}$ and $\mathbf{D}$, high density of inoculum. Curves describing change in the number of infected and susceptible roots and the proportion of infected roots in the absence of disease-induced growth (dotted lines) were produced using estimates of $r_{p}$ and $r_{s}$ obtained by fitting model 2 to microcosm data (Table 1$)$ with $f(I)=0$. These curves are compared with curves obtained by fitting model 2 to microcosm data $($ Table 1$)$ with $f(I)=\left[\left(\alpha_{1} I^{2}-\right.\right.$ $\left.\left.\alpha_{2} I\right) /\left(\alpha_{3}+I^{2}\right)\right]$ (solid lines).

A Epidemic dominated by primary infection

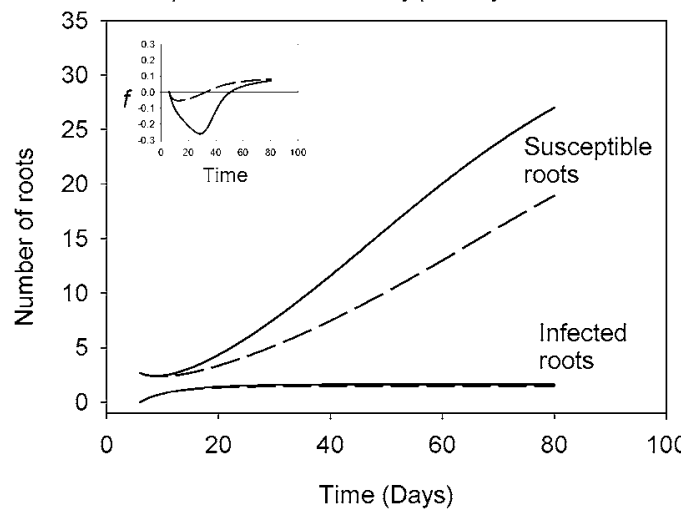

B Epidemic dominated by secondary infection

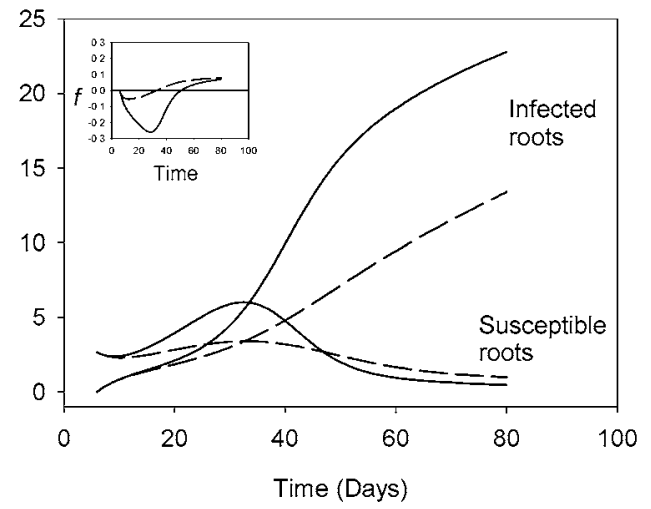

Fig. 6. The effect of extending the amplitude and duration of disease-induced root growth on epidemics dominated by $\mathbf{A}$, primary infection and $\mathbf{B}$, secondary infection. Curves (dotted) describing change in susceptible and infected roots over time were obtained from microcosm experiments where $f(I)=\left[\left(0.11 I^{2}-\right.\right.$ $\left.0.37 I) /\left(3.92+I^{2}\right)\right]$ giving and $f(t)$ inset (dotted line). These were compared with an extended response (solid lines) where $f(I)=\left[\left(0.25 I^{2}-4.0 I\right) /\left(30.0+I^{2}\right)\right]$ giving $f(t)$ inset (solid line). 
the host plant. This resulted in an average of no more than two additional roots per plant at either inoculum density (Fig. 2). We conjecture, however, that under conditions suboptimal for rapid spread of disease in the field, the amplitude and duration of increased root production may be prolonged. Model simulations show that this could lead to a pronounced response as the host reacts to a slower rate of infection (Fig. 6). The epidemiological consequences of this depend on the balance between primary and secondary infection. For epidemics dominated by primary infection, where levels of secondary infection are reduced by, for example, dry conditions later in the season, this may lead ultimately to an increase in the number of healthy roots (Fig. 6A). For epidemics dominated by secondary infection however, this form of host response will ultimately lead to pathogen invasion (Fig. 6B) (6).

Although we detected differences in stimulation of root production between epidemics initiated from low and high inoculum density during the early phase ( $<40$ days) of epidemics (Fig. 4), the consequence of this was only observed as differences in root number during the latter phase ( $>50$ days) of the epidemics (Fig. 2 ). This delayed response may well reflect the differing capacities of the seminal and adventitious root systems to respond to disease whereby infection of seminal roots stimulates overproduction of adventitious roots. The aim of this paper was not to examine differences in the contribution of seminal and adventitious roots to disease-induced root growth. However, differences in the growth and physiology of the two root systems are well documented (10, 11) and their precise role in response to disease and the epidemiology of take-all awaits further analysis. We suggest that the models introduced here will provide a useful starting point.

Using parameters estimated from the microcosm experiments, we predicted the contribution of disease-induced root growth to the spread of disease by comparing an imaginary cultivar that does not respond to disease with one that does (Fig. 5). Additional root production caused a marginal dilution of disease during the initial phase of the epidemic but stimulated the spread of disease during the second phase of the epidemic. We have shown elsewhere that small differences in primary infection can have a significant effect on final levels of disease and that changes in root (substrate) density may lead to thresholds for invasion of a pathogen into a growing root system (2). Others, using linear and descriptive models and single observations over time, have failed to find evidence that take-all significantly affects root production or the dynamics of disease in field-grown wheat (9). We propose that this is because the relationship is not simple, involving both stimulation and inhibition of root production, which under field conditions may occur at different times and in different places in response to differences in initial conditions (inoculum density) and at the scale of the individual plant. The sensitivity of our analysis was assisted by use of the microcosm experiments, which allow detailed, nondestructive measurements of disease and root dynamics in a way that is seldom practical in the field. Microcosms also permit strict control of environmental conditions so that the underlying host and pathogen dynamics can be teased out in the absence of large amounts of environmental variability that may obscure the relationships in the analysis of field observations. Nevertheless, even in the absence of environmental heterogeneity, such a nonlinear, dynamical response to differences in local inoculum density would lead to large differences in the relationship between root numbers and disease, rendering it undetectable by conventional analysis yet profoundly important as a mechanism for dictating the outcome of an epidemic.

\section{ACKNOWLEDGMENTS}

This work was co-funded by an award from the Biotechnology and Biological Sciences Research Council and by CPB Twyfords.

\section{LITERATURE CITED}

1. Bailey, D. J., and Gilligan, C. A. 1999. Dynamics of primary and secondary infection in take-all epidemics. Phytopathology 89:84-91.

2. Bailey, D. J., Otten, W., and Gilligan, C. A. 2000. Percolation, heterogeneity and the saprotrophic invasion of soil by the fungal plant pathogen Rhizoctonia solani. New Phytol. 146:535-544.

3. Chambers, J. C. 1971. Glasshouse studies on Ophiobolus graminis as a cause of whiteheads in wheat. Phytopathol. Z. 71:169-182.

4. Gilligan, C. A. 2002. An epidemiological framework for disease management. Adv. Bot. Res. 38:1-64.

5. Gilligan, C. A., Gubbins, S., and Simons, S. A. 1997. Analysis and fitting of an SIR model with host response to infection load for a plant disease. Philos. Trans. R. Soc. Lond. Ser. B. 352:353-364.

6. Gubbins, S., Gilligan, C. A., and Kleczkowski, A. 2000. Population dynamics of plant-parasite interactions: Thresholds for invasion. Theor. Popul. Biol. 57:219-233.

7. Hide, G. A., and Firmager, J. P. 1990. Effect of an isolate of Rhizoctonia solani Kuhn Ag8 from diseased barley on the growth and infection of potatoes. Potato Res. 33:229-234.

8. Manners, J. G., and Myers, A. 1981. Effects on growth and physiology. Pages 237-248 in: Biology and Control of Take-All. M. J. C. Asher and P. J. C. Shipton, eds. Academic Press, New York.

9. Penrose, L. D. J. 1995. 2 wheat genotypes differ in root disease due to Gaeumannomyces graminis without interaction with site. Soil Biol. Biochem. 27:133-138.

10. Sallans, B. J. 1942. The importance of various roots to the wheat plant. Sci. Agric. 23:17-26.

11. Simmonds, P. M., and Sallans, B. J. 1933. Further studies on amputations of wheat roots in relation to diseases of the root system. Sci. Agric. 13:439-448.

12. Skou, J. P. 1975. Studies on the take-all fungus Gaeumannomyces graminis. V. Development and regeneration of roots in cereal species during the attack. K. Vet. Landbohojsk. Arsskr. 42-160. 